\title{
Dynamic analysis of externally excited NES-controlled systems via a mixed Multiple Scale/Harmonic Balance algorithm
}

\author{
Angelo Luongo · Daniele Zulli
}

\begin{abstract}
A general, nonlinear, multi-d.o.f. structure, excited by harmonic external force in 1:1 resonance with one of the modes of the system, is considered. The structure is attached to an essentially nonlinear oscillator, with small mass and damping (Nonlinear Energy Sink, NES). The scope of the NES is to passively control the amplitude of vibrations of the main structure. A mixed Multiple Scale/Harmonic Balance Method (MSHBM) is proposed to get the differential equations describing the slow- and fast-flow dynamics of the whole structure. The main advantage of the procedure is that no complexification-averaging is required, so that the analysis is reconducted in the framework of the classical perturbation techniques.
\end{abstract}

Keywords Multiple scale method · Harmonic balance $\cdot$ Nonlinear energy sink $\cdot$ Strongly modulated response

\section{Introduction}

Nonlinear Energy Sinks (NES) are mechanical devices, constituted by strongly nonlinear oscillators, attached to a main structure. Their mass is generally small, compared to that of the main system, and their

A. Luongo $(\bowtie) \cdot$ D. Zulli

DISAT-University of L'Aquila, Via Giovanni Gronchi,

18,67100 L'Aquila, AQ, Italy

e-mail: angelo.luongo@univaq.it goal is to absorb energy and to dissipate it, as a passive control device, through targeted energy transfers (TET) (see $[1,2]$ for an extensive background).

TET is a physical phenomenon that allows to convey energy associated with vibrations in a one-way, irreversible fashion, from a donor to a receptor. It was deeply investigated in the literature in the analytical, numerical, and experimental sense [3-7].

The main feature of the NES, consequence of its essential nonlinear nature, consists in the capacity of oscillate at any frequencies, giving rise of large band tuning with the structure to be controlled. The existence of relaxation oscillations and strongly modulated responses (SMR) is an other typical feature of this kind of systems (referred as singular perturbation problems), where both slow and fast dynamics are naturally involved (see $[8,9]$ ).

Recently, these kind of devices have received great attention in the literature, being used in many applications. In $[10,11]$, a NES was applied to a main linear oscillator harmonically excited by a 1:1 resonant force. In $[12,13]$, multiple parallel NESs were considered to dissipate first-mode oscillations of a linear structure under impulse as well as harmonic forcing. In [14], nonsmooth NES was considered to control a two-d.o.f. system. In [15], NES was used to suppress aeroelastic instabilities on a rigid wing, modeled as a two-d.o.f. section-model, under stationary wind. In [16], a single NES is used to control oscillations of a long-span bridge prone to coupled flutter. 
convenient to introduce the relative displacement between main structure and NES, $z:=\mathbf{r}^{T} \mathbf{x}-y$, so that Eqs. (1) and (2) become:

$$
\begin{aligned}
& \mathbf{M} \ddot{\mathbf{x}}+\mathbf{C} \dot{\mathbf{x}}+\mathbf{K}(\sigma) \mathbf{x}+\xi \dot{z} \mathbf{r}+\kappa z^{3} \mathbf{r}+\mathbf{n}(\mathbf{x}, \mathbf{x}, \mathbf{x}) \\
& \quad=\mathbf{f} \cos \omega t \\
& m\left(\mathbf{r}^{T} \ddot{\mathbf{x}}-\ddot{z}\right)-\xi \dot{z}-\kappa z^{3}=0
\end{aligned}
$$

The dependent variables are rescaled through a nondimensional small parameter $\varepsilon>0$, as $(\mathbf{x}, z):=$ $\varepsilon^{1 / 2}(\tilde{\mathbf{x}}, \tilde{z})$, consistently with the presence of cubic nonlinearity. The damping is rescaled as $\mathbf{C}=\varepsilon \tilde{\mathbf{C}}$ and the external force as $\mathbf{f}=\varepsilon^{3 / 2} \tilde{\mathbf{f}}$, consistently with the idea to order both damping and excitation at the same level of the nonlinearity. The structural parameter $\sigma$ is rescaled as $\sigma=\varepsilon \tilde{\sigma}$. The parameters of the NES are also rescaled, since both its mass and damping are assumed small: $(m, \xi):=\varepsilon(\tilde{m}, \tilde{\xi})$. The rescaling and series expansion of $\mathbf{K}(\sigma)$ lead to the following equations, after omission of tilde and division by $\varepsilon^{1 / 2}$ :

$$
\begin{gathered}
\mathbf{M} \ddot{\mathbf{x}}+\varepsilon \mathbf{C} \dot{\mathbf{x}}+\left(\mathbf{K}_{0}+\varepsilon \sigma \mathbf{K}_{1}\right) \mathbf{x}+\varepsilon \xi \dot{z} \mathbf{r} \\
\quad+\varepsilon \kappa z^{3} \mathbf{r}+\varepsilon \mathbf{n}(\mathbf{x}, \mathbf{x}, \mathbf{x})=\varepsilon \mathbf{f} \cos \omega t \\
\varepsilon m\left(\mathbf{r}^{T} \ddot{\mathbf{x}}-\ddot{z}\right)-\varepsilon \xi \dot{z}-\varepsilon \kappa z^{3}=0
\end{gathered}
$$

where $\mathbf{K}_{0}:=\mathbf{K}(0)$ and $\mathbf{K}_{1}:=\partial \mathbf{K}(0) / \partial \sigma$.

According to the multiple scale method, independent time scales $t_{0}:=t, t_{1}:=\varepsilon t, t_{2}=\varepsilon^{2} t, \ldots$ are introduced and, consistently, the derivatives expressed as $\frac{d}{d t}=d_{0}+\varepsilon d_{1}+\varepsilon^{2} d_{2}+\cdots$ and $\frac{d^{2}}{d t^{2}}=d_{0}^{2}+2 \varepsilon d_{0} d_{1}+$ $\varepsilon^{2}\left(d_{1}^{2}+2 d_{0} d_{1}\right)+\cdots$. Moreover, the dependent variables are expanded in series as

$$
\left\{\begin{array}{l}
\mathbf{x} \\
z
\end{array}\right\}=\left\{\begin{array}{l}
\mathbf{x}_{0} \\
z_{0}
\end{array}\right\}+\varepsilon\left\{\begin{array}{l}
\mathbf{x}_{1} \\
z_{1}
\end{array}\right\}+\varepsilon^{2}\left\{\begin{array}{l}
\mathbf{x}_{2} \\
z_{2}
\end{array}\right\}+\cdots
$$

Substituting in Eqs. (5) and (6) and collecting terms of the same order in $\varepsilon$, lead to the following perturbation equations:

order $\varepsilon^{0}$ :

$$
\mathbf{M} d_{0}^{2} \mathbf{x}_{0}+\mathbf{K}_{0} \mathbf{x}_{0}=0
$$

order $\varepsilon^{1}$ :

$$
\begin{aligned}
\mathbf{M} d_{0}^{2} & \mathbf{x}_{1}+\mathbf{K}_{0} \mathbf{x}_{1} \\
= & -2 \mathbf{M} d_{0} d_{1} \mathbf{x}_{0}-\mathbf{C} d_{0} \mathbf{x}_{0}-\sigma \mathbf{K}_{1} \mathbf{x}_{0} \\
& \quad-\xi d_{0} z_{0} \mathbf{r}-\kappa z_{0}^{3} \mathbf{r}-\mathbf{n}\left(\mathbf{x}_{0}, \mathbf{x}_{0}, \mathbf{x}_{0}\right)+\mathbf{f} \cos \omega t_{0}
\end{aligned}
$$

$m\left(\mathbf{r}^{T} d_{0}^{2} \mathbf{x}_{0}-d_{0}^{2} z_{0}\right)-\xi d_{0} z_{0}-\kappa z_{0}^{3}=0$

order $\varepsilon^{2}$ :

$$
\begin{aligned}
\mathbf{M} & d_{0}^{2} \mathbf{x}_{2}+\mathbf{K}_{0} \mathbf{x}_{2} \\
= & -\mathbf{M}\left(d_{1}^{2} \mathbf{x}_{0}+2 d_{0} d_{2} \mathbf{x}_{0}+2 d_{0} d_{1} \mathbf{x}_{1}\right) \\
& \quad-\mathbf{C}\left(d_{2} \mathbf{x}_{0}+d_{1} \mathbf{x}_{1}\right)-\sigma \mathbf{K}_{1} \mathbf{x}_{1}-\xi\left(d_{0} z_{1}+d_{1} z_{0}\right) \mathbf{r} \\
& \quad-3 \kappa z_{0}^{2} z_{1} \mathbf{r}-3 \mathbf{n}\left(\mathbf{x}_{1}, \mathbf{x}_{0}, \mathbf{x}_{0}\right) \\
m\left(\mathbf{r}^{T}\right. & \left.d_{0}^{2} \mathbf{x}_{1}-d_{0}^{2} z_{1}\right)-\xi d_{0} z_{1}-3 \kappa z_{0}^{2} z_{1} \\
= & 2 m\left(d_{0} d_{1} z_{0}-\mathbf{r}^{T} d_{0} d_{1} \mathbf{x}_{0}\right)+\xi d_{1} z_{0}
\end{aligned}
$$

It should be noticed that, because of the vanishingly small values of the mass and damping, as well of the lack of linear stiffness, no equation of motion relevant to NES appears in the generator problem (order $\varepsilon^{0}$ ), which therefore describes the linear dynamics of the main structure alone (as if NES were disengaged).

The 1:1 external resonance with just one of the linear modes of the main system, together with the presence of damping, entails that the remaining nonresonant modes bring a higher-order contribution to the overall response. Therefore, only the contribution related to the resonant mode is retained in the solution of Eq. (8), i.e.,

$\mathbf{x}_{0}\left(t_{0}, t_{1}, \ldots\right)=A\left(t_{1}, \ldots\right) \mathbf{u} e^{i \omega t_{0}}+c c$

where: $A\left(t_{1}, \ldots\right)$ is a complex modal amplitude, whose modulation on the slower time-scales must be evaluated; $i$ is the imaginary unit; $i \omega$ and $\mathbf{u}$ are the resonant eigenvalue and (real) eigenvector of the problem $\left(\mathbf{K}_{0}+\lambda^{2} \mathbf{M}\right) \mathbf{u}=\mathbf{0}$, respectively; finally $c c$ stands for complex conjugate.

The $\varepsilon$-order perturbation equations (9) and (10) are now addressed, and the NES equation (10) considered first. Since its (steady) solution cannot be expressed by elementary (nor Jacobi) functions, the harmonic balance method is used, letting

$z_{0}\left(t_{0}, t_{1}, \ldots\right)=B_{1}\left(t_{1}, \ldots\right) e^{i \omega t_{0}}+c c$

where $B_{1}\left(t_{1}, \ldots\right)$ is a (first order) slowly modulated complex amplitude, to be evaluated. Other frequency components $(3 \omega, 5 \omega, \ldots)$ are not considered here (see the next Sect. 4 for a discussion). Equations (13) and (14) are substituted in Eq. (10) and only $\omega$-frequency terms are balanced. This step leads to

$-m \omega^{2}\left(B_{1}-r A\right)+i \xi \omega B_{1}+3 \kappa B_{1}^{2} \bar{B}_{1}=0$

where $r:=\mathbf{r}^{T} \mathbf{u}$. 
Equation (15) provides, at the first order of perturbation, an algebraic constrain between the (active) resonant amplitude of oscillation of the main structure and the (passive) amplitude of the NES elongation, $B_{1}$; it, therefore, describes a codimension- 2 manifold in the state-space, on which the asymptotic dynamics take place. To get the (first order) real form of the manifold, the expressions $A(t):=\frac{1}{2} a(t) e^{i \alpha(t)}$ and $B_{1}(t):=\frac{1}{2} b(t) e^{i \beta(t)}$, are substituted in Eq. (15) and real and imaginary parts separated:

$m \omega^{2} r a \cos (\alpha-\beta)=m \omega^{2} b-\frac{3}{4} \kappa b^{3}$

$m \omega^{2} r a \sin (\alpha-\beta)=-\xi \omega b$

These latter, once squared and summed, provide

$m^{2} \omega^{4} r^{2} a^{2}=\left(m \omega^{2} b-\frac{3}{4} \kappa b^{3}\right)^{2}+\xi^{2} \omega^{2} b^{2}$

which is a constrain between the two involved real amplitudes. Equation (15) (or (18)), however, is unable to describe motions which occur (in relaxation oscillation) externally to the manifold, which typically consist in very fast jumps. To capture this effect, a further perturbation equation must be tackled. Equation (9) is then considered, in which $z_{0}$ is assumed as in Eq. (14). By requiring that the resonant forcing term is orthogonal to the eigenvector $\mathbf{u}$ (solvability condition), the following condition is obtained:

$$
\begin{aligned}
d_{1} A= & \left(c_{1}+i \sigma c_{2}\right) A+c_{3} B_{1}+i c_{4} B_{1}^{2} \bar{B}_{1} \\
& +i c_{5} A_{1}^{2} \bar{A}_{1}+i c_{6} f
\end{aligned}
$$

where $f:=\mathbf{u}^{T} \mathbf{f}$, and the expressions of the coefficients $c_{j}$ are given in Appendix. By substituting Eq. (19) in Eq. (9) and by solving for $\mathbf{x}_{1}$, it holds

$$
\begin{aligned}
\mathbf{x}_{1}= & i A \mathbf{w}_{1} e^{i \omega t_{0}}+\sigma A \mathbf{w}_{2} e^{i \omega t_{0}}+B_{1} \mathbf{w}_{3} e^{i \omega t_{0}} \\
& +B_{1}^{2} \bar{B}_{1} \mathbf{w}_{4} e^{i \omega t_{0}}+A^{2} \bar{A} \mathbf{w}_{5} e^{i \omega t_{0}}+\mathbf{w}_{6} e^{i \omega t_{0}} \\
& +B_{1}^{3} \mathbf{w}_{7} e^{3 i \omega t_{0}}+A^{3} \mathbf{w}_{8} e^{3 i \omega t_{0}}+c c
\end{aligned}
$$

where $\mathbf{w}_{j},(j=1, \ldots, 8)$ are defined in Appendix.

Equation (12) is finally considered: a new harmonic balance is carried out, assuming the following expression for $z_{1}$ :

$z_{1}\left(t_{0}, t_{1}, \ldots\right)=B_{2}\left(t_{1}, \ldots\right) e^{i \omega t_{0}}+c c$
Substituting Eqs. (13), (14), and (20) in Eq. (12) and balancing the $\omega$-frequency terms, the following equation is obtained:

$$
\begin{aligned}
& -m \omega^{2} B_{2}+i \xi \omega B_{2}+3 \kappa B_{1}^{2} \bar{B}_{2}+6 \kappa B_{1} \bar{B}_{1} B_{2} \\
& \quad+(\xi+2 i m \omega) d_{1} B_{1}-2 i m \omega r d_{1} A \\
& +m \omega^{2}\left(i A w_{1}+\sigma A w_{2}+i B_{1} w_{3}+B_{1}^{2} \bar{B}_{1} w_{4}\right. \\
& \left.\quad+A^{2} \bar{A} w_{5}\right)+m \omega^{2} w_{6}=0
\end{aligned}
$$

where $w_{j}:=\mathbf{r}^{T} \mathbf{w}_{j}, j=1, \ldots, 6$. Equations (15) and (22) can be reconstituted, using the definition $B:=$ $B_{1}+\varepsilon B_{2}$, and coming back to the true time, to obtain:

$$
\begin{aligned}
(\xi+ & 2 i m \omega) \dot{B}-2 i m \omega r \dot{A} \\
= & \left(m \omega^{2}\left(1-i w_{3}\right)-i \xi \omega\right) B \\
& -\left(3 \kappa+m \omega^{2} w_{4}\right) B^{2} \bar{B}-m \omega^{2}\left(r+i w_{1}+\sigma w_{2}\right) A \\
& -m \omega^{2} w_{5} A^{2} \bar{A}-m \omega^{2} w_{6}
\end{aligned}
$$

It appears that this equation now describes the dynamics of the amplitude $B$, differently from Eq. (15). The key-term containing $\dot{B}$ comes out only at the secondorder, since it is affected by small coefficients $\xi$ and $m$, thus revealing the nature of singular perturbation. In contrast, the term proportional to $\dot{A}$, which also appears at this order, does not add any qualitative new contributions, being ruled by Eq. (19).

If the perturbation procedure is truncated at order $\varepsilon$ for the main system equation, the solvability condition (19) can be written in terms of the true time:

$\dot{A}=\left(c_{1}+i \sigma c_{2}\right) A+c_{3} B+i c_{4} B^{2} \bar{B}+i c_{5} A^{2} \bar{A}+i c_{6} f$

To get the polar form of Eqs. (24) and (23), $A(t):=$ $\frac{1}{2} a(t) e^{i \alpha(t)}$ and $B(t):=\frac{1}{2} b(t) e^{i \beta(t)}$ are substituted in them and real and imaginary parts separated, thus obtaining (from Eq. (24)):

$$
\begin{array}{rl}
\dot{a}=c_{1} & a+c_{3} b \cos (\alpha-\beta)+\frac{c_{4}}{4} b^{3} \sin (\alpha-\beta) \\
a \dot{\alpha}= & \sigma c_{2} a+c_{3} b \sin (\alpha-\beta)+\frac{c_{4}}{4} b^{3} \cos (\alpha-\beta) \\
& +\frac{c_{5}}{4} a^{3}+c_{6} f
\end{array}
$$

and (from Eq. (23)) 


$$
\begin{aligned}
\xi \dot{b}- & 2 \omega m b \dot{\beta}+2 \omega m r(\dot{a} \sin (\alpha-\beta)+a \dot{\alpha} \cos (\alpha-\beta)) \\
= & m \omega^{2} w_{1} a \sin (\alpha-\beta) \\
& -m \omega^{2}\left(r+\sigma w_{2}\right) a \cos (\alpha-\beta)+m \omega^{2} b \\
& -\left(m \omega^{2} w_{4}+3 \kappa\right) \frac{b^{3}}{4}-m \omega^{2} w_{5} \frac{a^{3}}{4} \cos (\alpha-\beta) \\
& -m \omega^{2} w_{6} \cos \beta
\end{aligned}
$$

$$
\begin{aligned}
2 \omega m \dot{b} & +\xi b \dot{\beta}-2 \omega m r(\dot{a} \cos (\alpha-\beta)-a \dot{\alpha} \sin (\alpha-\beta)) \\
= & -m \omega^{2} w_{1} a \cos (\alpha-\beta) \\
& -m \omega^{2}\left(r+\sigma w_{2}\right) a \sin (\alpha-\beta) \\
& -\left(m \omega^{2} w_{3}+\omega \xi\right) b-m \omega^{2} w_{5} \frac{a^{3}}{4} \sin (\alpha-\beta) \\
& +m \omega^{2} w_{6} \sin \beta
\end{aligned}
$$

The nonlinear singular perturbation problem Eqs. (25)-(28) describes the dynamics of the main system attached to the NES, in terms of the real dependent variables $a, b, \alpha, \beta$. Its equilibrium points, determined taking $\dot{a}=\dot{b}=\dot{\alpha}=\dot{\beta}=0$, represent periodic oscillations in the displacement $\mathbf{x}, z$.

\section{Sample systems and numerical results}

A sample system, already studied in $[2,11]$, is considered here to check the reliability of the method presented in Sect. 2. A complete study of the dynamics of the proposed example, as well as of the possible beneficial effect of the NES, however, are not fulfilled herein, since they are out of the aim of this paper.

The main system consists of a 1-d.o.f. linear undamped system, with attached NES, a sketch of which is shown in Fig. 2. The nondimensional equations of motion are:

$\ddot{x}+\left(\omega^{2}+\sigma\right) x-\xi(\dot{y}-\dot{x})-\kappa(y-x)^{3}=f \cos \omega t$

$m \ddot{y}+\xi(\dot{y}-\dot{x})+\kappa(y-x)^{3}=0$

that, for $z:=x-y$, become:

$\ddot{x}+\left(\omega^{2}+\sigma\right) x-\xi \dot{z}-\kappa z^{3}=f \cos \omega t$

$m(\ddot{z}-\dot{x})+\xi \dot{z}+\kappa z^{3}=0$

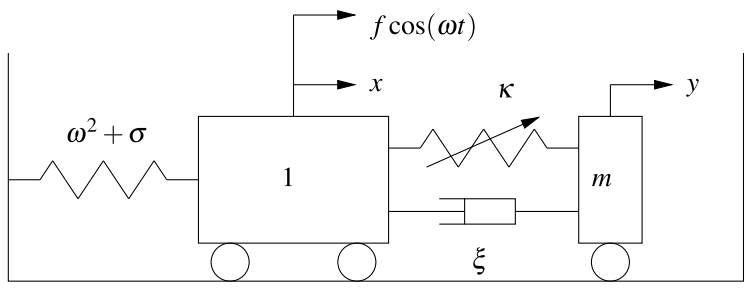

Fig. 2 Sketch of a analyzed system

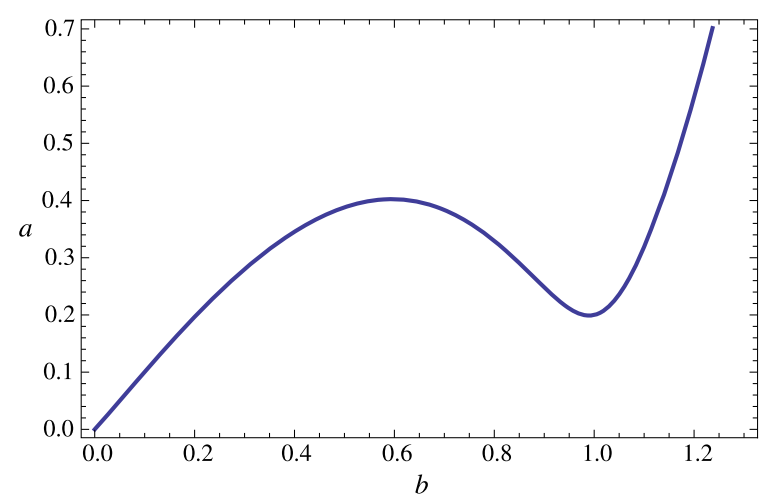

Fig. 3 Nonlinear manifold when $m=0.05, \xi=0.01$, $\kappa=0.067, \omega=1$

Therefore, comparing it with Eqs. (3) and (4), it results:

$$
\begin{aligned}
& N=1, \quad \mathbf{x}=x, \quad \mathbf{M}=1, \quad \mathbf{C}=0, \\
& \mathbf{K}_{0}=\omega^{2}, \quad \mathbf{K}_{1}=1, \quad \mathbf{n}(\mathbf{x}, \mathbf{x}, \mathbf{x})=0, \\
& \mathbf{f}=f
\end{aligned}
$$

The nonlinear manifold, Eq. (18), becomes

$$
\left(\frac{3 \kappa b^{2}}{8 m \omega}+\frac{\omega b}{2}\right)^{2}+\left(\frac{\xi b}{2 m}\right)^{2}-\frac{\omega^{2} a^{2}}{4}=0
$$

and it is shown in Fig. 3 for $m=0.05, \xi=0.01$, $\kappa=0.067, \omega=1$ (which is the set of numerical values considered in $[2,11])$.

The (first order) solvability condition reads:

$\dot{A}=\frac{i \sigma}{2 \omega} A-\frac{\xi}{2} B-\frac{3 i \kappa}{2 \omega} B^{2} \bar{B}-\frac{i f}{4 \omega}$

and the (second order) harmonic balance becomes:

$$
\begin{aligned}
& 2 i m \omega \dot{A}-(2 i m \omega+\xi) \dot{B} \\
& \quad=m \omega^{2} A+\left(i \xi \omega-m \omega^{2}\right) B+3 \kappa B^{2} \bar{B}
\end{aligned}
$$


In polar form, they assume the forms:

$$
\dot{a}=\frac{3 k b^{3} \sin (\alpha-\beta)}{8 \omega}-\frac{1}{2} \xi b \cos (\alpha-\beta)-\frac{f \sin \alpha}{2 \omega}
$$

$$
\begin{aligned}
a \dot{\alpha}= & \frac{a \sigma}{2 \omega}+\frac{3 b^{3} k \cos (\alpha-\beta)}{8 \omega}+\frac{1}{2} b \xi \sin (\alpha-\beta) \\
& -\frac{f \cos \alpha}{2 \omega}
\end{aligned}
$$

and

$$
\begin{gathered}
m \dot{a} \sin (\alpha-\beta)+m a \dot{\alpha} \cos (\alpha-\beta)+\frac{\xi}{2 \omega} \dot{b}-m b \dot{\beta} \\
=-\frac{m \omega}{2} a \cos (\alpha-\beta)-\frac{3 b^{3} k}{8 \omega}+\frac{m \omega}{2} b
\end{gathered}
$$

$m \dot{a} \cos (\alpha-\beta)-m a \dot{\alpha} \sin (\alpha-\beta)-m \dot{b}-\frac{\xi}{2 \omega} b \dot{\beta}$

$$
=\frac{\xi}{2} b+\frac{m \omega}{2} a \sin (\alpha-\beta)
$$

When the NES is disengaged, since the main system is linear, the amplitudes of the periodic solutions in $x$ become

$a_{e}=\frac{f}{\sigma}$

$\tan \alpha_{e}=\frac{a_{e} \sigma}{2 \omega}$

which are always stable. They are the equilibrium points of Eqs. (37), (38), when $b=0$. Due to the lack of damping in the main system, the amplitude tends to infinite when $\sigma$ goes to zero.

In the following analysis, the NES is considered engaged. The branches of equilibrium points of the dynamical system (37)-(40), which represent periodic oscillations in the original variables $x$ and $z$, are shown in Fig. 4, for increasing values of $f$ (in Fig. 4a when $f=0.020$, in Fig. 4b when $f=0.039$ and in Fig. 4c when $f=0.075)$. The figures are obtained via the software AUTO [22]. In the same figures, some equilibrium points are marked by colored points, to get reference with other subsequent figures, while black boxes represent Hopf bifurcation points. It can be observed that multiple solutions exist in some intervals of $\sigma$, as in Fig. 4a around $\sigma=-0.10$ or $\sigma=0.10$. In Fig. $4 \mathrm{~b}$, around $\sigma=0.2$, three solutions exist and two of them are stable (green and yellow points, stable; red point, unstable); on the other hand, around the value $\sigma=-0.15$, three solutions exist and only one of them is stable (no colored points). A higher amplitude branch also exists, describing a closed island (only partially shown in the Figs. $4 \mathrm{a}, \mathrm{b}$ ). As the force is increased, the island gets wider and closer to the lower branch, until it disappears by merging with the lower branch (Fig. 4c). All these results are consistent with those shown in $[2,11]$.

Strongly modulated responses (SMR) are detected by numerical integration of the system (37)-(40). They represent quasiperiodic relaxation oscillations in the variables $a$ and $b$, typically describing cycles around the two folds of the nonlinear manifold shown in Fig. 3. They are triggered in dependence of the position of the equilibrium points.

When $\sigma=0.015, f=0.02, m=0.05, \xi=0.01$, $\kappa=0.067$, the equilibrium point is unique, unstable, and it is represented by the yellow point in Fig. 4a. In Fig. 5a, the same equilibrium point is shown (yellow point) on the nonlinear manifold (blue line), as well as the trajectory of the relaxation oscillation, describing an annular region (red line), while in Fig. 5b the latter is substituted by its Poincaré section (black points). The corresponding time evolutions of the amplitudes $a, b$ (obtained by numerical integration of Eqs. (37)-(40)) and of the reconstituted displacements $x, z$ are shown in Figs. 6a, b. They are in very good agreement with the solutions obtained by numerical integration of the original equations (31), (32), shown in Fig. 6c.

When $\sigma=0.2, f=0.039, m=0.05, \xi=0.01$, $\kappa=0.067, \omega=1$ (see Fig. 4b), the three different equilibrium points are marked with colored points. They are also shown in Fig. 7, lying on the nonlinear manifold. One of them is unstable (red point) and the other two are stable (green and yellow points). Two different trajectories are also shown Fig. 7 (magenta and black lines), asymptotically falling to the stable equilibria. Relaxation oscillations are not triggered and, therefore, periodic solution in $x, z$ are obtained.

When $\sigma=-0.3, f=0.075, m=0.05, \xi=0.01$, $\kappa=0.067, \omega=1$ (see Fig. 4c), the three different equilibrium points are marked and only the green one is stable. They are also shown in Fig. 8, on the nonlinear manifold. A relaxation oscillation is triggered, and its Poincaré section is shown (magenta points). For initial conditions close to the stable equilibrium point, 

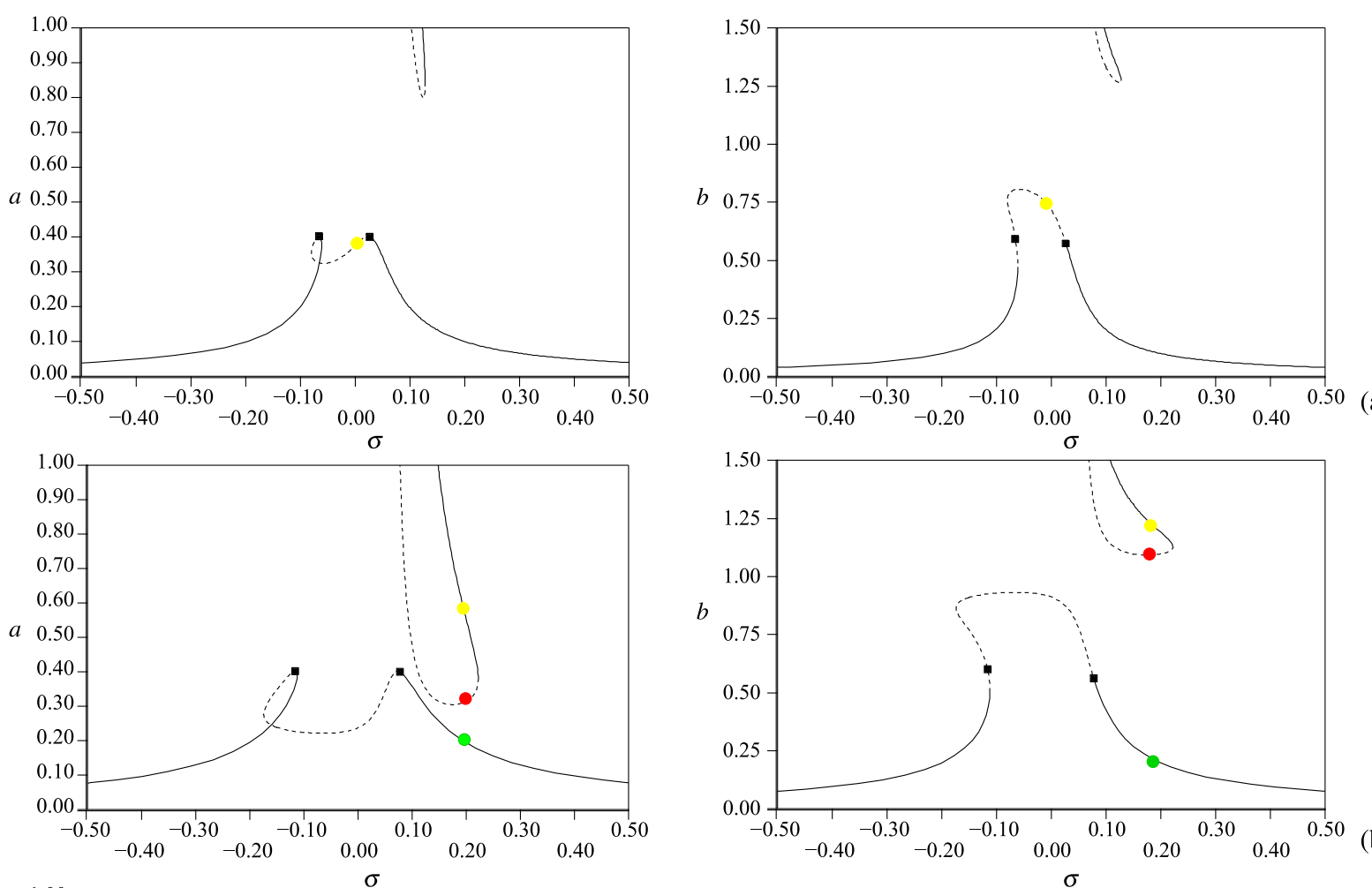

(b)
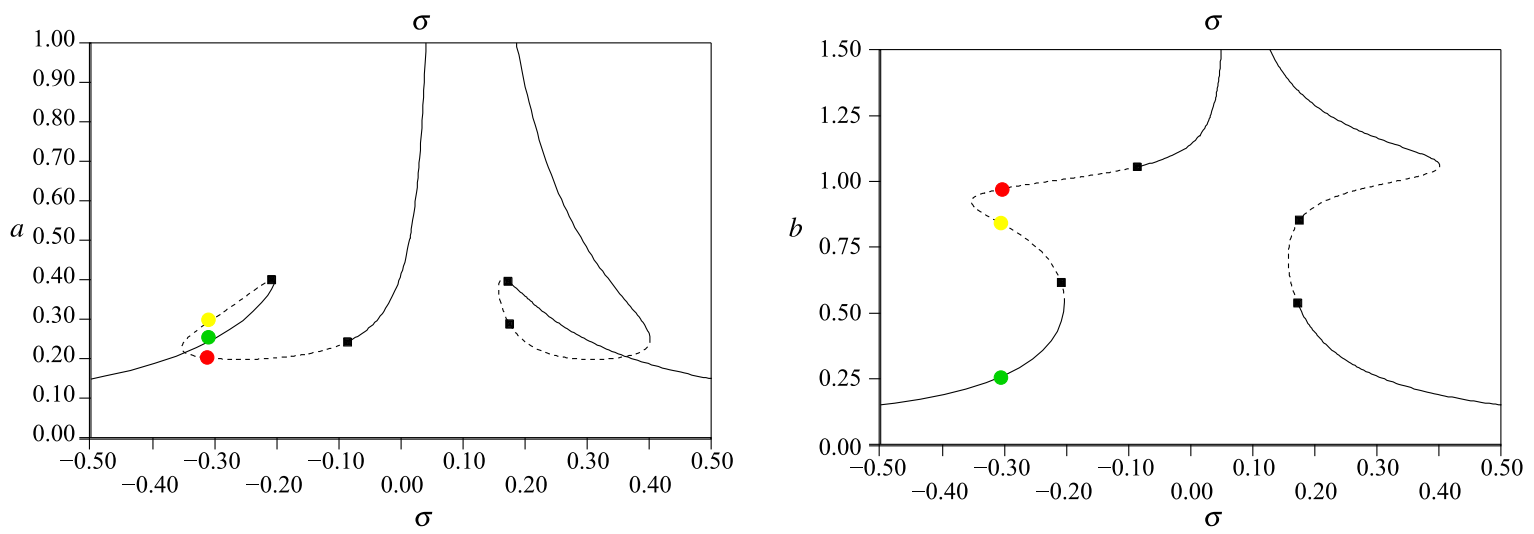

Fig. 4 Amplitudes $a$ and $b$ when NES is engaged, when $m=0.05, \xi=0.01, \kappa=0.067, \omega=1$, and (a) $f=0.020$, (b) $f=0.039$, (c) $f=0.075$. The filled squares indicate Hopf

bifurcation points. The colored points are equilibria referred to following figures. Continuous line: stable; dashed line: unstable

$f=0.01$. The unique equilibrium point (yellow point) is stable and stands on the right branch of the nonlinear manifold. Also, trajectories falling to the equilibrium points are found, obtained when initial conditions close to the equilibrium point are considered. They are not reported in the figure. The SMR quasiperiodic time evolutions of $a, b$, and corresponding quasi-periodic time evolutions of $x, z$ are shown in Figs. $11 \mathrm{a}, \mathrm{b}$, respectively. Again, they are in good agreement with the 


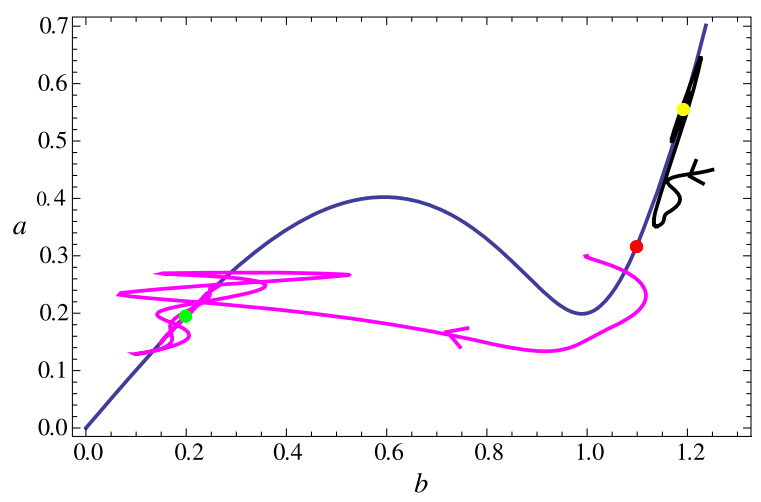

Fig. 7 Nonlinear manifold (blue line), three equilibrium points (green, red, and yellow points) and two transitional motions (magenta and black lines) falling to the equilibria, when $\sigma=0.2, f=0.039, m=0.05, \xi=0.01, \kappa=0.067, \omega=1$

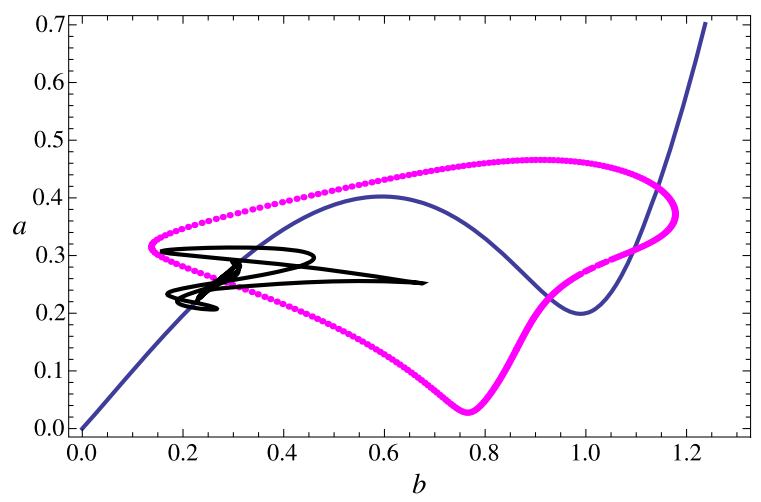

Fig. 8 Nonlinear manifold (blue line), three equilibrium points (red, green and yellow points), Poincaré map of the SMR response (magenta points), and transitional motion (black line) falling to the equilibrium point, when $\sigma=-0.3, f=0.075$, $m=0.05, \xi=0.01, \kappa=0.067, \omega=1$

solution of the original equations (31), (32), shown in Fig. 11c.

\section{Discussion}

Few specific topics are now addressed, concerning the potentialities of the algorithm illustrated herein, namely, (a) the influence of higher harmonics, neglected in solving the NES equation, and (b) the analytical investigation of SMR, carried out in literature via a different approach.

\subsection{Higher frequency-terms}

The choice of using just one frequency-term, namely the one with frequency $\omega$, in the Harmonic Balance (i.e., in Eqs. (14), (21)), might appear as a strong limitation of the procedure introduced herein. In principle, indeed, terms of frequency $3 \omega, 5 \omega, \ldots$ could be involved and become significant.

To solve this issue, as a fist attempt, terms of frequency $3 \omega$ have been considered, also using

$$
\begin{aligned}
& z_{0}\left(t_{0}, t_{1}, \ldots\right) \\
& \quad=B_{11}\left(t_{1}, \ldots\right) e^{i \omega t_{0}}+B_{13}\left(t_{1}, \ldots\right) e^{3 i \omega t_{0}}+c c \\
& z_{1}\left(t_{0}, t_{1}, \ldots\right) \\
& \quad=B_{21}\left(t_{1}, \ldots\right) e^{i \omega t_{0}}+B_{23}\left(t_{1}, \ldots\right) e^{3 i \omega t_{0}}+c c
\end{aligned}
$$

instead of Eqs. (14), (21), and obtaining corresponding equations which balance terms of frequency $\omega$ and $3 \omega$, instead of Eqs. (15), (22), not reported here.

It turns out that the amplitudes of terms of frequency $3 \omega$ are always of higher order than those of frequency $\omega$, and then resulting as negligible. They do not give any significant contribution to the dynamics of the system. In particular, after the reconstitution procedure $\left(B_{1}:=B_{11}+\varepsilon B_{21}, B_{3}:=B_{13}+\varepsilon B_{23}\right.$, and coming back to the true time), if $b_{1}$ is the real amplitude of $B_{1}$ and $b_{3}$ is the real amplitude of $B_{3}$, the manifold should be naturally pictured as a curve in the three-dimensional space $\left(b_{1}, b_{3}, a\right)$. For the numerical example considered in Sect. 3, it is shown in Fig. 12. The projection in the $\left(b_{1}, a\right)$-plane is practically the same of Fig. 3 whereas, looking at the projection in the $\left(b_{1}, b_{3}\right)$-plane, it can be observed that $b_{3}$ is much smaller than $b_{1}$ and $a$ in the considered range. Corresponding results (not reported here) show that the effect of higher frequency $3 \omega$ is negligible in the equilibrium branches and SMR, also.

In any case, the presence of higher frequencyterms would preserve the structure of singular perturbation system, since Eqs. (24), (23) would be modified into:

$$
\begin{aligned}
\dot{A} & =f(A, \mathbf{B}) \\
\varepsilon \dot{\mathbf{B}} & =\mathbf{g}(A, \mathbf{B})
\end{aligned}
$$

where $\mathbf{B}=\left\{B_{1}, B_{3}\right\}$.

\subsection{Analytical detection of SMR}

The analytical detection of the SMR can be accomplished with the outcomes of the MSHBM, as in the framework of the Manevitch complexification. This task is based on the study of the singularities of the 

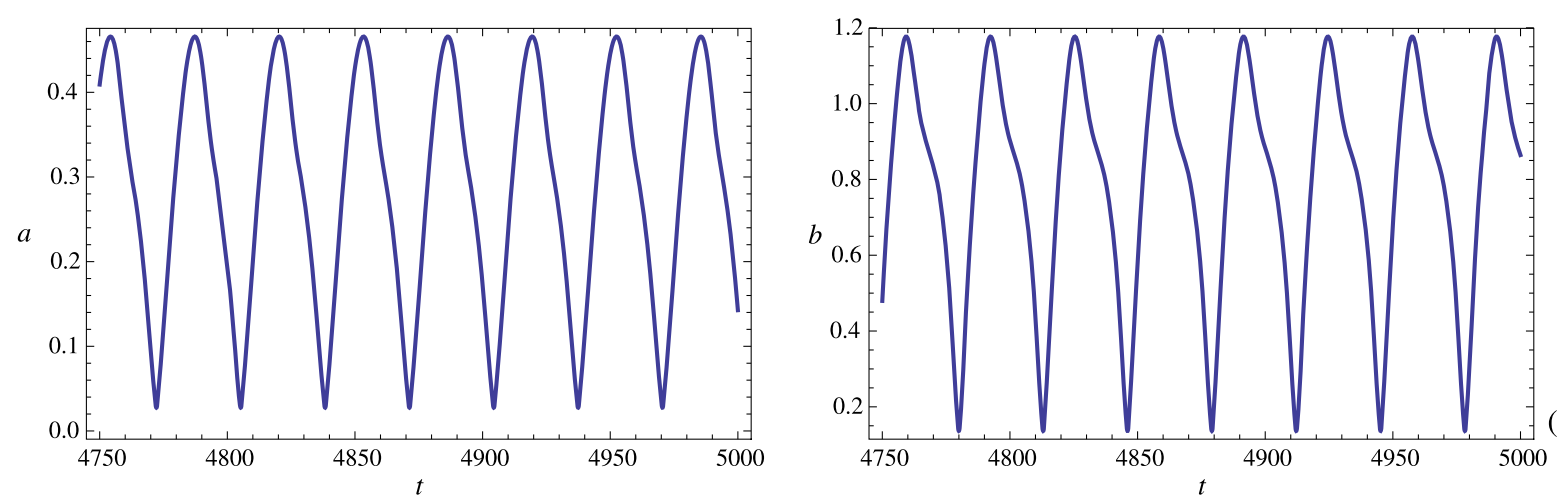

(a)
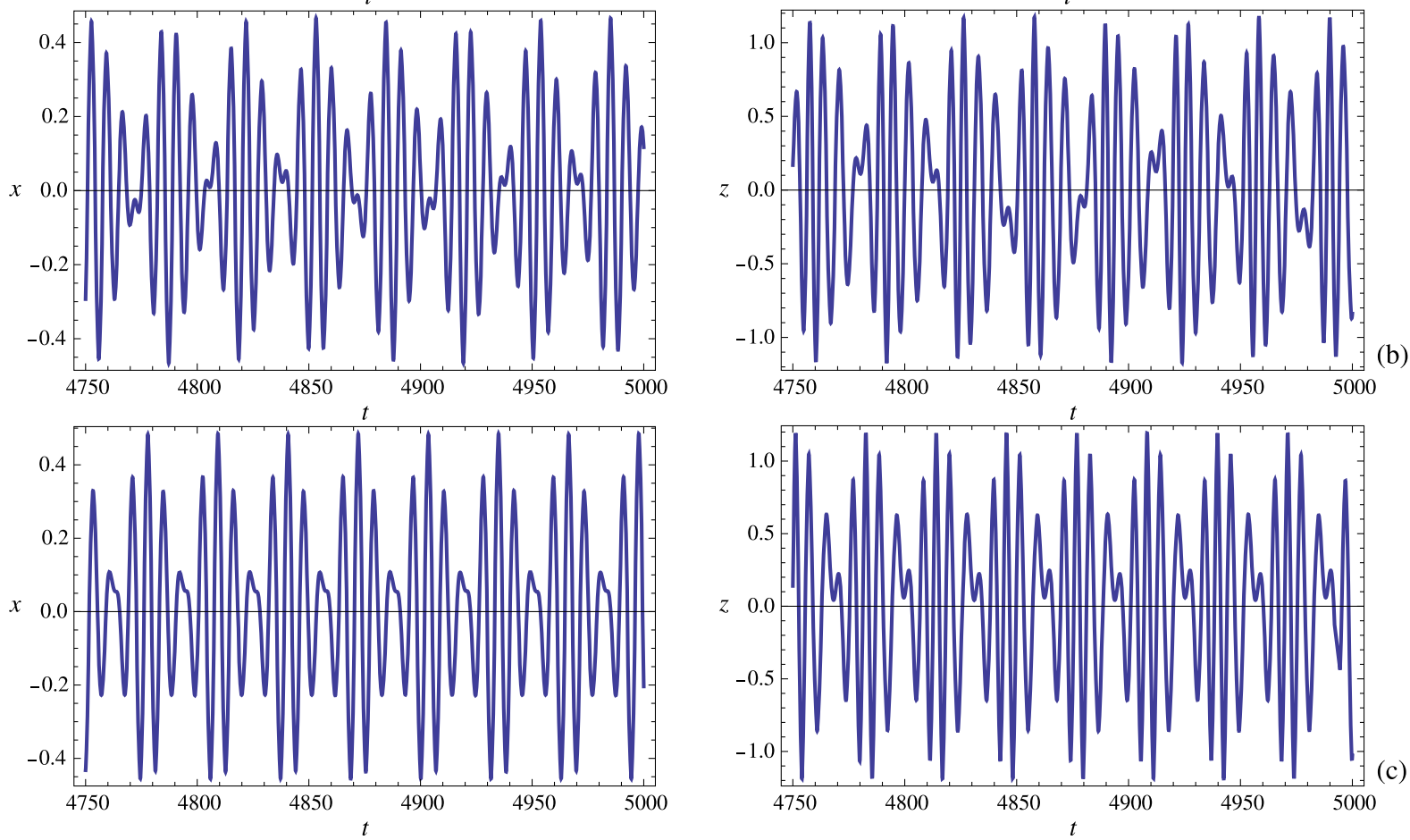

Fig. 9 SMR, when $\sigma=-0.3, f=0.075, m=0.05, \xi=0.01, \kappa=0.067, \omega=1$; (a) amplitudes by MSHBM; (b) reconstituted response; (c) numerical integration of the original equations (31), (32)

slow dynamics on the manifold (see [11]). Already at the first perturbation step of the MSHBM, the slow dynamics on the manifold are addressed, whereas the second perturbation step allows the study of the dynamics also outside the manifold. In fact, if Eq. (15) is solved to obtain the expression

$A=B_{1}-\frac{i \xi B_{1}}{m \omega_{1}}-\frac{3 k B_{1}^{2} \bar{B}_{1}}{m \omega_{1}^{2}}$

and it is substituted in Eq. (19), after coming back to the true time, the following complex differential equa- tion in the dependent variables $B_{1}, \bar{B}_{1}$ is obtained:

$$
\begin{gathered}
\dot{B}_{1}\left(-\frac{6 k B_{1} \bar{B}_{1}}{m \omega^{2}}-\frac{i \xi}{m \omega}+1\right)-\frac{3 k B_{1}^{2} \dot{\bar{B}}_{1}}{m \omega^{2}} \\
=\frac{i f}{4 \omega}+B_{1}^{2} \bar{B}_{1}\left(\frac{3 i k \sigma}{2 m \omega^{3}}-\frac{3 i k}{2 \omega}\right) \\
+B_{1}\left(-\frac{\xi \sigma}{2 m \omega^{2}}+\frac{\xi}{2}-\frac{i \sigma}{2 \omega}\right)
\end{gathered}
$$

Using the polar representation $B_{1}(t)=\frac{1}{2} b(t) e^{i \beta(t)}$, and separating real and imaginary parts, two real dif- 


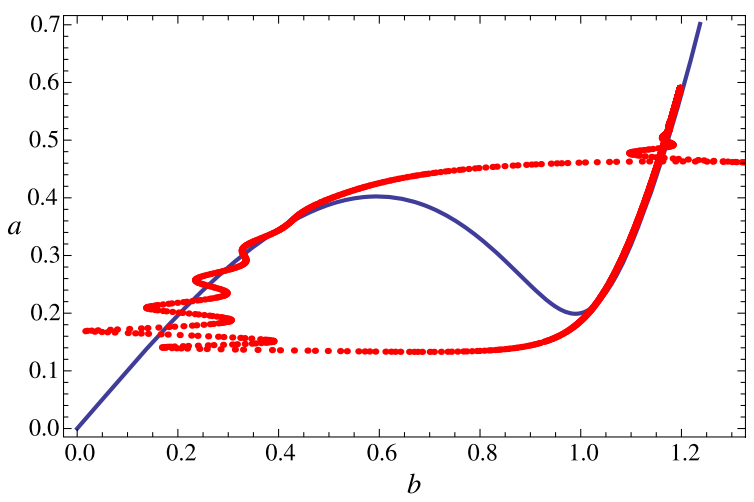

Fig. 10 Nonlinear manifold (blue line), equilibrium point (yellow point) and Poincaré map of the SMR response (red points), when $\sigma=-0.015, f=0.01, m=0.005, \xi=0.001$, $\kappa=0.0067, \omega=1$
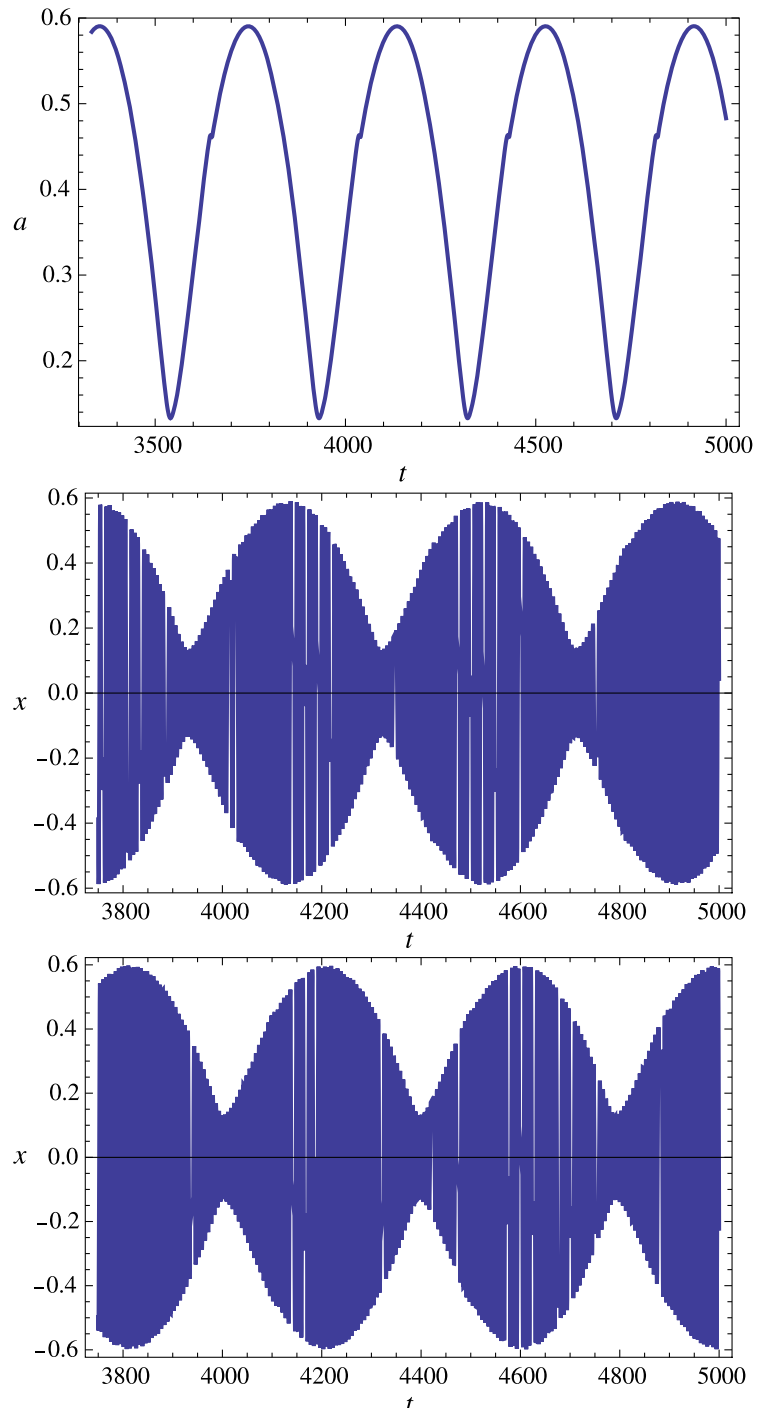

ferential equations in $b(t), \beta(t)$ are obtained. The phase portrait in the stable sides of the manifold, representing $b(t)$ vs. $\beta(t)$, is shown in Fig. 13 for the numerical example, when $\sigma=0.15, f=0.02$, $m=0.05, \xi=0.01, \kappa=0.067, \omega=1$, where the limits of existence of the SMR, corresponding to the phases $\beta_{1}$ and $\beta_{2}$, are drawn by the vertical dashed lines.

\section{Conclusions}

In this paper, a general, nonlinear, multi-d.o.f. system, equipped with an essentially nonlinear oscillator with

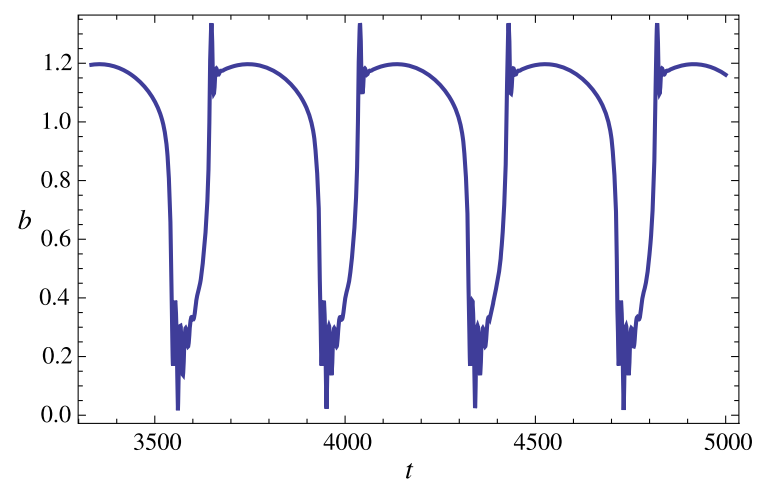

(a)

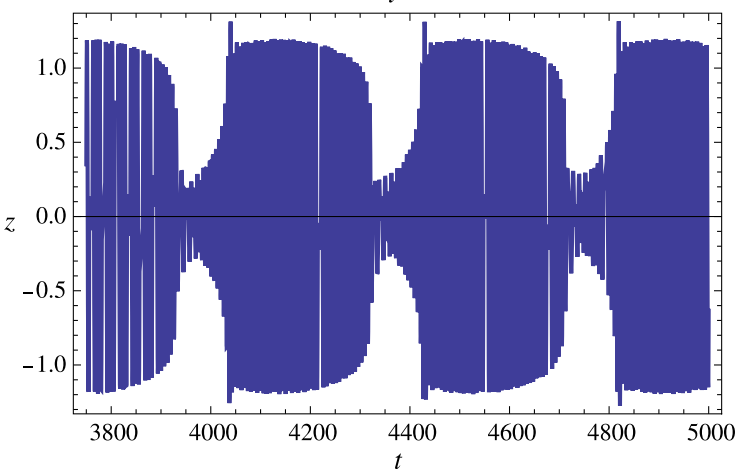

(b)

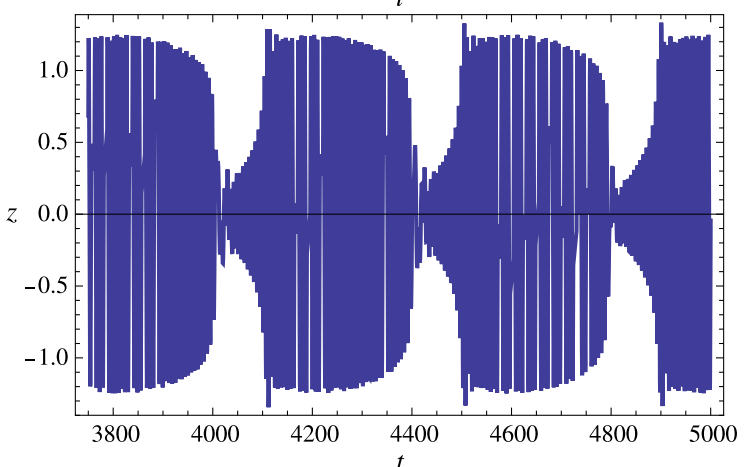

Fig. 11 SMR, when $\sigma=-0.015, f=0.01, m=0.005, \xi=0.001, \kappa=0.0067, \omega=1 ;$ (a) amplitudes by MSHBM; (b) reconstituted response; (c) numerical integration of the original equations (31), (32) 
monic forcing and frequency detuning. J. Sound Vib. 315, 746-765 (2008)

1. Vakakis, A.F., Gendelman, O.V., Bergman, L.A., McFarland, D.M., Kerschen, G., Lee, Y.S.: Nonlinear Targeted Energy Transfer in Mechanical and Structural Systems I. Springer, New York (2008a)

2. Vakakis, A.F., Gendelman, O.V., Bergman, L.A., McFarland, D.M., Kerschen, G., Lee, Y.S.: Nonlinear Targeted Energy Transfer in Mechanical and Structural Systems II. Springer, New York (2008b)

3. Maniadis, P., Kopidakis, G., Aubry, S.: Classical and quantum targeted energy transfer between nonlinear oscillators. Physica D 188, 153-177 (2004)

4. Kerschen, G., Kowtko, J.J., McFarland, D.M., Bergman, L.A., Vakakis, A.F.: Theoretical and experimental study of multimodal targeted energy transfer in a system of coupled oscillators. Nonlinear Dyn. 47, 285-309 (2007)

5. Panagopoulos, P.N., Gendelman, O., Vakakis, A.F.: Robustness of nonlinear targeted energy transfer in coupled oscillators to changes of initial conditions. Nonlinear Dyn. 47, 377-387 (2007)

6. Aubry, S., Kopidakis, G., Morgante, A.M., Tsironis, G.P.: Analytic conditions for targeted energy transfer between nonlinear oscillators or discrete breathers. Physica B 296, 222-236 (2001)

7. Tsakirtzis, S., Panagopoulos, P.N., Kerschen, G., Gendelman, O., Vakakis, A.F., Bergman, L.A.: Complex dynamics and targeted energy transfer in linear oscillators coupled to multi-degree-of-freedom essentially nonlinear attachments. Nonlinear Dyn. 48, 285-318 (2007)

8. Guckenheimer, J., Hoffman, K., Weckesser, W.: Bifurcations of relaxation oscillations near folded saddles. Int. J. Bifurc. Chaos 15, 3411-3421 (2005)

9. Guckenheimer, J., Wechselberger, M., Young, L.-S.: Chaotic attractors of relaxation oscillators. Nonlinearity $\mathbf{1 9}$, 701-720 (2006)

10. Gendelman, O.V., Starosvetsky, Y., Feldman, M.: Attractors of harmonically forced linear oscillator with attached nonlinear energy sink i: description of response regimes. Nonlinear Dyn. 51, 31-46 (2008)

11. Starosvetsky, Y., Gendelman, O.V.: Response regimes of linear oscillator coupled to nonlinear energy sink with har-
12. Vaurigaud, B., Savadkoohi, A.T., Lamarque, C.-H.: Targeted energy transfer with parallel nonlinear energy sinks. Part I: design theory and numerical results. Nonlinear Dyn. 66(4), 763-780 (2011a)

13. Savadkoohi, A.T., Vaurigaud, B., Lamarque, C.-H., Pernot, S.: Targeted energy transfer with parallel nonlinear energy sinks. Part II: theory and experiments. Nonlinear Dyn. 67(1), 37-46 (2012)

14. Lamarque, C.-H., Gendelman, O.V., Savadkoohi, A.T., Etcheverria, E.: Targeted energy transfer in mechanical systems by means of non-smooth nonlinear energy sink. Acta Mech. 221, 175-200 (2011)

15. Gendelman, O.V., Vakakis, A.F., Bergman, L.A., McFarland, D.M.: Asymptotic analysis of passive nonlinear suppression of aeroelastic instabilities of a rigid wing in subsonic flow. SIAM J. Appl. Math. 70(5), 1655-1677 (2010)

16. Vaurigaud, B., Manevitch, L.I., Lamarque, C.-H.: Passive control of aeroelastic instability in a long span bridge model prone to coupled flutter using targeted energy transfer. J. Sound Vib. 330, 2580-2595 (2011b)

17. Manevitch, L.: The description of localized normal modes in a chain of nonlinear coupled oscillators using complex variables. Nonlinear Dyn. 25, 95-109 (2001)

18. Gendelman, O.V.: Targeted energy transfer in systems with non-polynomial nonlinearity. J. Sound Vib. 315, 732-745 (2008)

19. Nayfeh, A.H., Mook, D.T.: Nonlinear Oscillations. Wiley, New York (1979)

20. Jiang, X., McFarland, D.M., Bergman, L.A., Vakakis, A.F.: Steady state passive nonlinear energy pumping in coupled oscillators: theoretical and experimental results. Nonlinear Dyn. 33, 87-102 (2003)

21. Malatkar, P., Nayfeh, A.H.: Steady-state dynamics of a linear structure weakly coupled to an essentially nonlinear oscillator. Nonlinear Dyn. 47, 167-179 (2007)

22. Doedel, E.J., Oldeman, B.E.: AUTO-07P: continuation and bifurcation software for ordinary differential equation (2012). URL http://cmvl.cs.concordia.ca/auto/ 